\title{
Barreras para la atención en salud durante el diagnóstico y tratamiento del cáncer gástrico
}

\author{
Obstacles to Health Care During the Diagnosis and Treatment of \\ Gastric Cancer
}

\section{Barreiras na atencão e nos cuidados da saúde para o diagnóstico e tratamento do câncer gástrico}

\author{
Angélica María Amado-Niño, Ft., MSc. * \\ Leydi Yurani Mantilla-Villabona. ** \\ Diana Carolina Ospina-Galeano. ** \\ Dely María Auxiliadora Maturana-Martínez. *** \\ Claudia Janeth Uribe-Pérez, MD. ****
}

\begin{abstract}
Resumen
Introducción: El cáncer gástrico es una neoplasia maligna de alta morbimortalidad. Ocupa el quinto lugar en prevalencia mundial. Objetivo: Describir las barreras para la atención en salud durante el diagnóstico y tratamiento del cáncer gástrico. Metodología: Para la revisión de la literatura se emplearon bases de datos (PubMed, Lilacs, Ebsco, Science-direct, Ovid y google académico), que incluyó la consulta de ensayos clínicos, revisiones de tema y guías de manejo relacionadas con cáncer gástrico y sus barreras durante el diagnóstico y tratamiento, entre 20092016. Resultados: 56 artículos cumplieron con los criterios de inclusión y exclusión. Las barreras halladas son
\end{abstract}

\begin{abstract}
administrativas, institucionales, económicas, culturales y de conocimientos del paciente y el médico, que limitan el acceso a la atención médica. Conclusión: En Colombia, la fragmentación y segmentación del sistema de salud impone barreras en la atención de los adultos con cáncer gástrico ocasionando las disparidades en salud que impactan en su sobrevida. [Amado-Niño AM, Mantilla-Villabona LY, OspinaGaleano DC, Maturana-Martínez DMA, Uribe-Pérez CJ. Barreras para la atención en salud durante el diagnóstico y tratamiento del cáncer gástrico. MedUNAB 2017; 19(3): 211-220]
\end{abstract}

Palabras clave: Neoplasias Gástricas; Servicios de Salud; Calidad de la Atención de Salud; Factores Socioeconómicos; Accesibilidad a los Servicios de Salud; Factores de Riesgo.

* Fisioterapeuta, Magíster en Salud Pública. Docente cátedra. Universidad Industrial de Santander, Bucaramanga, Santander, Colombia.

** Estudiante programa de Medicina XII semestre. Facultad de Ciencias de la Salud, Universidad Autónoma de Bucaramanga, Floridablanca, Santander, Colombia.

*** Estudiante programa de Medicina X semestre. Facultad de Ciencias de la Salud, Universidad Autónoma de Bucaramanga, Floridablanca, Santander, Colombia.

****Médica patóloga, Dermatopatóloga. Directora Registro Poblacional de Cáncer del Área Metropolitana de Bucaramanga. Docente en Facultad de Ciencias de la Salud, UniversidadAutónoma de Bucaramanga, Floridablanca, Santander, Colombia.

Correspondencia: Claudia Janeth Uribe-Pérez, Registro Poblacional de Cáncer del Área Metropolitana de Bucaramanga, Centro de Investigaciones Biomédicas, Facultad de Ciencias de la Salud, Universidad Autónoma de Bucaramanga, Calle 157 19-55 Campus El Bosque, Cañaveral, Floridablanca, Santander, Colombia. Correo electrónico: curibep@unab.edu.co. 


\section{Abstract}

Introduction: Gastric cancer is a malignant neoplasm of high morbi-mortality. It ranks fifth in world prevalence. Objective: To describe the obstacles to health care during the diagnosis and treatment of gastric cancer. Methodology: PubMed, Lilacs, Ebsco, Science-direct, Ovid and google scholar were used to the review of literature that included the consultation of clinical trials, topic reviews and management guides related to gastric cancer and its barriers during diagnosis and treatment between 2009 and 2016. Results: 56 articles achieved the inclusion and exclusion criteria. The obstacles found are administrative, institutional, economic, cultural and knowledge of the patient and the physician about gastric cancer which could limit the access to medical care. Conclusion: In Colombia, the fragmentation and segmentation of the health system impose obstacles on the care of adults with gastric cancer, which cause disparities in health that impact on their survival. [Amado-Niño $A M$, Mantilla-Villabona LY, Ospina-Galeano DC, MaturanaMartínez DMA, Uribe-Pérez CJ. Obstacles to health care during the diagnosis and treatment of gastric cancer. MedUNAB 2017; 19(3): 211-220]

Keywords: Stomach Neoplasms; Health Services; Quality of Health Care; Socioeconomic Factors; Health Services Accessibility; Risk Factors.

\section{Introducción}

El Cáncer gástrico $(\mathrm{CG})$ es una neoplasia maligna, generada por factores de riesgo genéticos, infecciosos causados por Helicobacter pylori (1); y dietarios debido al consumo de alimentos ahumados, ricos en nitritos, entre otros (2-4). La incidencia del CG en el mundo es de 952,000 casos al año en ambos géneros y más del $70 \%$ de los casos $(677,000)$ ocurren en países en vía de desarrollo; en ambos sexos, ocupa el quinto lugar en prevalencia y es la tercera causa de muerte asociada con el cáncer en el mundo ( 8.9 por 100,000 habitantes); la mayor tasa de mortalidad por CG se ha reportado en Asia Oriental (24 hombres y 9.8 mujeres por 100,000 habitantes), seguida por Europa central y del Este, América Latina, y la tasa más baja se ha reportado en América del Norte (2.8 hombres y 1.5 mujeres por 100,000 habitantes) $(5,6)$.

El CG es un problema de salud pública en Colombia, debido al aumento en su incidencia y mortalidad en las últimas décadas y al impacto que este genera a nivel socioeconómico en quien lo padece y su familia (7). Según GLOBOCAN, Colombia, en el año 2012 el CG fue la segunda causa incidente de morbilidad, después del cáncer de próstata en hombres y la cuarta causa para las mujeres, luego del cáncer de mama, cuello uterino y colo-rectal; con una incidencia para ambos sexos de 13.4 por 100,0000 habitantes (6).

\section{Resumo}

Introdução: O câncer gástrico é uma neoplasia maligna de alta morbidade e mortalidade. Ele ocupa o quinto lugar na prevalência mundial. Objetivo: Descrever as barreiras encontradas no sistema de saúde para o diagnóstico e o tratamento do câncer gástrico. Metodologia: Para rever a literatura existente foi utilizada a base de dados da (PubMed, Lilacs, EBSCO, Science-diretas, Ovídio e Google acadêmico), incluindo a consulta dos ensaios clínicos, a revisão do tema e as guias relacionadas com câncer gástrico e suas barreiras durante o diagnóstico e tratamento, entre 2009-2016. Resultados: 56 artigos preencheram os critérios de inclusão e de exclusão. As barreiras encontradas são de carater administrativo, institucionais, económicas, culturais e do conhecimento do paciente e do médico, limitando assim o acesso aos cuidados médicos. Conclusão: Na Colômbia, a fragmentação e segmentação do sistema de saúde impõe barreiras no atendimento dos adultos com câncer gástrico causando as disparidades de saúde que afetam a sua sobrevivência. [Amado-Niño AM, Mantilla-Villabona LY, Ospina-Galeano DC, Maturana-Martínez DMA, Uribe-Pérez CJ. Barreiras na atencão e nos cuidados da saúde para o diagnóstico e tratamento do câncergástrico. MedUNAB 2017; 19(3): 211-220]

Palavras-chave: Neoplasias Gástricas; Serviços de Saúde; Qualidade da Assistência à Saúde; Fatores Socioeconômicos; Acesso aos Serviços de Saúde; Fatores de Risco.

Ahora bien, en el Área Metropolitana de Bucaramanga (AMB), el CG es la segunda causa incidente de enfermedad por cáncer, después del cáncer de próstata; y es la cuarta en mujeres, después de cáncer de mama, cérvix y colo-rectal; con tasas de 22.4 y 10.3 por 100,000 habitantes, respectivamente. Sumado a esto, la mortalidad por 100,000 habitantes para ambos sexos durante 2003-2007 fue de 14.2; para hombres fue de 20.1 y para mujeres 9.9; con ello, constituye la primera causa de muerte en el sexo masculino y la segunda causa en el femenino, después del cáncer de mama (8).

El comportamiento del CG en Colombia y en el AMB sugiere que existen dificultades para su detección temprana y tratamiento (9), algunas originadas por la segmentación institucional y fragmentación operativa propia del Sistema General de Seguridad Social en Salud (SGSSS) que funciona bajo una lógica de mercado, con un modelo de aseguramiento que responde a dos regímenes: el contributivo (personas con capacidad de pago) y el subsidiado para la población sin recursos económicos. Dicho sistema está basado en la competencia regida por las Empresas Administradoras de Planes de Beneficios (EAPB), encargadas de gestionar el riesgo y organizar su propia red de prestadores que proporcionen servicios de salud continuos, integrales, eficientes y coordinados entre los diferentes niveles de atención requeridos por un usuario sin presentar barreras o conflictos $(10,11)$. 
Dicha segmentación poblacional, fragmentación operativa e intermediación entre las EAPB y los prestadores de los servicios de salud genera inequidades en el uso de los servicios, entre las que destacan: menor acceso en el régimen subsidiado y los no asegurados para los servicios de atención primaria y especializada respecto al régimen contributivo (12); negación de la atención; interposición de acciones de tutela como vía legítima para el acceso a los servicios; prolongación del sufrimiento y deterioro del estado de salud del paciente y su familia, entre otros (13). Esos factores lesionan el principio de equidad horizontal, porque determinan que el uso de los servicios de salud dependa del régimen y no de las necesidades del paciente, independientemente de factores socioeconómicos o demográficos(14); así, estas limitaciones ocasionan disparidades tan profundas que no se superan con el aumento de la cobertura y se requieren acciones intersectoriales(14).

No obstante, una amplia cobertura y la articulación de las instituciones involucradas puede disminuir la evolución de las barreras (15), las cuales afectan el seguimiento del proceso de salud-enfermedad, ocasionan el reinicio en el procedimiento de diagnóstico, retrasan el tratamiento y cambios en la mortalidad según los determinantes sociales del paciente $(16,17)$. Por lo anterior, la revisión sobre la literatura se propone identificar las barreras para la atención en salud, durante el diagnóstico y tratamiento, del cáncer gástrico en el mundo y en Colombia, Se ha de tener en cuenta que no existen otras revisiones acerca de este tema a nivel nacional.

\section{Metodología}

Este artículo es una revisión de la literatura indexada en PubMed, LILACS (Literatura Latinoamericana y del Caribe en Ciencias de la Salud) Ebsco, Science direct, Ovid y Google Académico que permitió responder a la pregunta ¿Cuáles son las barreras para la atención en salud, durante el diagnóstico y tratamiento del cáncer gástrico, en el mundo y en Colombia?, para adelantar la búsqueda se siguió la estrategia PICO: P: adultos con cáncer gástrico; I: diagnóstico y tratamiento del CG; C: Barreras para la atención en salud según el sistema de salud en el mundo y en Colombia; O: Impacto de las barreras en los adultos con CG. Apoyada por palabras claves en inglés como: "Stomach Neoplasms"; "Health Services"; "Quality of Health Care"; "Socioeconomic Factors"; "Health Services Accessibility"; "Risk Factors" y en español se usaron sus equivalentes. Se incluyeron: ensayos clínicos, revisiones de tema y guías de manejo relacionadas con barreras y/o inequidades para la atención en salud del cáncer gástrico durante su diagnóstico y tratamiento; publicados en inglés o español desde el año 2009 hasta 2016, disponibles en texto libre y completo.

Se excluyeron los artículos publicados antes del año 2009 y aquellos que describieran barreras en la atención en salud respecto a un tipo de cáncer específico diferente al cáncer gástrico, así como los que abordaron factores genéticos o biomoleculares clínicos referentes al diagnóstico y tratamiento de la enfermedad, aparición de un segundo cáncer y los que relataran la atención en salud clínica de pacientes con cáncer metastásico. También aquellos publicados en otros idiomas diferentes al inglés o al español, junto con la literatura gris.

Para la selección de los artículos tres de los autores realizaron una búsqueda conjunta que permitió, en común acuerdo, obtener la información que se tomaría en cuenta para la revisión, al atender los criterios planteados de inclusión y exclusión, que posteriormente fue leída y analizada de manera individual, sin presentarse discrepancias entre los aportes o conceptos emitidos por cada una de las lectoras. En consecuencia, con la estrategia de búsqueda propuesta se identificaron preliminarmente 443 artículos; luego se eliminaron los duplicados, además, después de la revisión de títulos y abstract se excluyeron 387. Como resultado 56 artículos fueron descargados en texto completo para llevar a cabo la presente revisión.

\section{Resultados}

El análisis de la literatura permitió definir que las barreras para la atención en salud del cáncer gástrico durante el diagnóstico y tratamiento, en el mundo y en Colombia, están orientadas por obstáculos administrativos, institucionales, económicos, culturales y de conocimiento, tanto del personal de salud como del paciente que impactan en el estado de salud del adulto con cáncer gástrico, en su calidad de vida y en la de su familia. (Tabla 1).

\section{Barreras administrativas}

La presencia de barreras administrativas se refleja en la alteración de la oportunidad y continuidad para el diagnóstico y tratamiento, es decir, fallas en la atención integral y coordinada, como: la dilatación del tiempo entre la emisión de las órdenes médicas y su cumplimiento, debido a trámites excesivos para la aprobación de procedimientos y medicamentos(18). Lo anterior se asocia con el significado y los sentimientos que el adulto con cáncer gástrico y su familia construyen de la enfermedad, pues se percibe como un factor que empeora su condición médica, reduce sus posibilidades de recuperación completa y puede conducir a la muerte(13).

Uno de los factores que facilita la aparición de las barreras administrativas es el tipo de seguridad social. En Estados Unidos, los adultos con cáncer con cobertura diferente al Medicaid tienen mayor acceso al tratamiento (79.6\% cirugía radical o radioterapia) y sobrevida a los dos años (86.2\%) en contraste con quienes tienen cobertura Medicaid (67.9\% tratamiento y $69.1 \%$ sobrevida) o no están 
Tabla 1. Barreras para la atención en salud del cáncer gástrico durante su diagnóstico y tratamiento.

\section{BARRERAS ADMINISTRATIVAS}

\begin{tabular}{ll}
\hline \multicolumn{1}{c}{ Durante el diagnóstico del CG } & \multicolumn{1}{c}{ Durante el tratamiento del CG } \\
\hline Contratación entre EAPBa e IPS & Tiempo en trámites y autorizaciones. \\
- Interrupción de contratos con las IPS. & - Tiempo prolongado en trámites para la remisión a \\
- Contratos a corto plazo y bajos salarios para & otras instituciones o a otro profesional. \\
el personal de atención sanitaria. & - Trámites prolongados para la aprobación de \\
Atención en nivel primario: & procedimientos y medicamentos.
\end{tabular}

- Competencias básicas para la identificación temprana del $\mathrm{CG}^{\mathrm{c}}$

- Falta de coordinaciones en la red de atención al adulto con cáncer gástrico.

Estructura y operatividad del sistema:

- Tipo de cobertura del sistema de salud

\section{Acceso a la red de atención en salud y oferta:}

- Poca accesibilidad a instalaciones especializadas.

- IPS presenta oferta de servicios que no corresponde a la realidad efectiva de atención.

- Oferta de horas de atención que excede las que se pueden cubrir con el personal existente en oncología clínica, hematología y gastroenterología entre otras.

- Negación del acceso a la atención en salud.

- Pérdida de continuidad y oportunidad de la atención.

- Duplicación de las pruebas diagnósticas.

- Retraso en el diagnóstico del cáncer.

- Sentimientos negativos frente a la enfermedad y asociación de muerte cercana.

- Dificultad en la comunicación médico paciente ante un evento de alto contenido emocional y de impacto físico como es el cáncer.

- Discapacidad por alteración del rol laboral y dedicación exclusiva a trámites para conseguir la atención en salud.

- Polifarmacia

- Reducción de las opciones terapéuticas y de la posibilidad de recuperación.

- Mortalidad temprana en CG ydisminución de la sobrevida.

\section{BARRERAS INSTITUCIONALES}

\begin{tabular}{|c|c|c|}
\hline $\begin{array}{l}\text { Características en la oferta y acceso real a los } \\
\text { servicios de salud: } \\
\text { - Oportunidad en la atención } \\
\text { - Carente red de prestadores de servicios de salud } \\
\text { para cribado, diagnóstico y tratamiento. }\end{array}$ & $\begin{array}{l}\text { - Pericia médica y el volumen de pacientes } \\
\text { atendidos. } \\
\text { - Disponibilidad de camas hospitalarias y unidades } \\
\text { de cuidado oncológico y paliativo. }\end{array}$ & $\begin{array}{l}\text { - Retraso del diagnóstico. } \\
\text { - Disminución de la sobrevida } \\
\text { - Deterioro de la calidad de vida } \\
\text { - Acciones de tutela }\end{array}$ \\
\hline \multicolumn{2}{|c|}{$\begin{array}{l}\text { Aumento del gasto de bolsillo (pagos directos que destinan las familias para solventar los requerimientos de } \\
\text { la atención de la salud) por el costo de la atención en salud, los traslados, hospedaje, alimentación del adulto } \\
\text { enfermo y su cuidador. }\end{array}$} & $\begin{array}{l}\text { - Deserción laboral, pérdida del empleo o } \\
\text { disminución de la capacidad de generar ingresos. } \\
\text { - Diagnóstico en estadío avanzado }\end{array}$ \\
\hline $\begin{array}{l}\text { - Nivel de ingresos insuficiente frente a los gastos } \\
\text { en salud. }\end{array}$ & $\begin{array}{l}\text { - Acceso a otros profesionales y opciones } \\
\text { terapéuticas }\end{array}$ & $\begin{array}{l}\text { - Abandono o pérdida de la oportunidad en el } \\
\text { tratamiento curativo y paliativo } \\
\text { - Pobreza } \\
\text { - Aumento de la mortalidad }\end{array}$ \\
\hline \multicolumn{3}{|l|}{ BARRERAS CULTURALES } \\
\hline $\begin{array}{l}\text { - Creencias } \\
\text { - Percepciones y significados frente a la alteración } \\
\text { del estado de salud y su relación con cáncer. } \\
\text { - Familiares solicitan al médico ocultar el } \\
\text { diagnóstico al adulto enfermo. }\end{array}$ & $\begin{array}{l}\text { - Falta de apoyo psicosocial para el paciente y su } \\
\text { familia. } \\
\text { - } \quad \text { Rechazo y negación al tratamiento } \\
\text { - Dificultad para la comprensión del tratamiento y } \\
\text { pronóstico }\end{array}$ & $\begin{array}{l}\text { - Fallas en la comunicación médico paciente } \\
\text { - Demora en búsqueda de atención } \\
\text { - Angustia, preocupación, depresión, sentimiento de muerte } \\
\text { - Falta de adherencia y control del tratamiento o su abandono } \\
\text { - Aumento de la mortalidad } \\
\text { - Falta de entendimiento para la toma conjunta de decisiones. } \\
\text { - Cuidado paliativo como única opción terapéutica por } \\
\text { diagnóstico tardío. }\end{array}$ \\
\hline \multicolumn{3}{|l|}{ BARRERAS DE CONOCIMIENTOS } \\
\hline $\begin{array}{l}\text { - Emisión de diagnósticos diferentes al CG por la } \\
\text { inespecificidad de síntomas. } \\
\text { - La pericia médica para relacionar los signos y } \\
\text { síntomas inespecíficos con el CG como impresión } \\
\text { diagnóstica. } \\
\text { - Falta de conocimiento de la población sobre el CG. }\end{array}$ & $\begin{array}{l}\text { - Tratamientos sintomáticos sin seguimiento ni el } \\
\text { uso de medios diagnósticos. } \\
\text { - Desconocimiento de las opciones terapéuticas y } \\
\text { su pronóstico. }\end{array}$ & $\begin{array}{l}\text { - Aumento en la automedicación. } \\
\text { - Pérdida de la confianza en el sistema de salud } \\
\text { - Mecanismos incorrectos de remisión y contra remisión } \\
\text { - Retraso del diagnóstico y tratamiento. } \\
\text { - Disminución de sobrevida }\end{array}$ \\
\hline
\end{tabular}

a: EAPB: Empresas Administradoras de Planes de Beneficios; b: IPS: Institución Prestadora de Salud; c: Cáncer Gástrico

Fuente: Elaboración propia de los autores 
asegurados $(62.1 \%$ tratamiento y $66.5 \%$ cobertura). Estos resultados son multifactoriales y pueden estar conectados con la presencia de otras barreras, como la falta de acceso a medios de transporte, pobre apoyo social, barreras financieras, además, del estadío en que se encuentre la enfermedad y el tipo de cáncer(19).

En Colombia, un estudio demostró que el tipo de cobertura del sistema de salud y el estrato socioeconómico impactan en la sobrevida de los pacientes con cáncer gástrico; puesto que, los usuarios del régimen especial mostraron 11.6 meses de sobrevida comparado con quienes estaban afiliados al régimen contributivo ( 6.8 meses) y 4.5 meses en régimen subsidiado y 0 meses en pacientes no afiliados (9). En cuanto al factor socioeconómico, se evidenció que tienen mayor tiempo de sobrevida los pacientes con alto nivel económico (8.6 meses) frente aquellos con un bajo nivel económico (4.9 meses), en razón a que la mayoría de adultos con altos recursos financieros pagan un seguro médico privado, con el fin de disminuir los retrasos en las remisiones a especialistas o a la emisión de las órdenes para el tratamiento (9).

Otro factor considerado como barrera administrativa es la posibilidad de acceder a atención general y especializada para el estudio de los signos y síntomas relacionados al diagnóstico de CG; con el objetivo de evaluar si la infraestructura sanitaria impacta en el retraso en el diagnóstico y, como consecuencia, en la supervivencia de los pacientes con CG; en Alemania, a partir de la densidad de médicos generales y gastroenterólogos por cada 100 kilómetros cuadrados, se realizó un estudio donde se estableció que los médicos generales y gastroenterólogos se concentran en áreas urbanas y esto reduce el retraso en el diagnóstico de los pacientes asentados en las ciudades. Asimismo, la disponibilidad de atención por gastroenterología en áreas rurales aumenta la probabilidad de diagnóstico oportuno en un 48\% (20). Esto sugiere la necesidad de disponer de recurso humano especialista en gastroenterología como una alternativa para contrarrestar el diagnóstico tardío de CG y mejorar su sobrevida (20); sumado al seguimiento de pautas y protocolos nacionales para el tratamiento del CG que incluyan mayores unidades diagnósticas para el cáncer gástrico y recurso humano multidisciplinario para el abordaje integral(21). Al respecto, algunos autores describieron que los factores con mayor impacto favorable en la sobrevida de los adultos con cáncer gástrico son el estadío de la enfermedad al momento del diagnóstico, la oportunidad en el tratamiento quirúrgico y el acceso a instituciones especializadas(22).

Lo anterior, basado en la competencia administrada, incentiva, en el caso de Colombia, que las EAPB establezcan contratos a corto plazo y compren servicios de una manera fragmentada. Así, un único episodio de la enfermedad se divide en múltiples momentos de atención de tiempo reducido con diferentes proveedores, lo cual dificulta la transferencia de información clínica, la comunicación y la colaboración directa entre los proveedores de servicios de salud (16). Estas barreras ocasionan pérdida de la credibilidad en la respuesta organizada del sistema de salud frente a la necesidad sentida de la población, así como la identificación de diferencias entre los regímenes de atención en individuos con necesidades equivalentes determinadas a los mecanismos de financiación de los planes de beneficios(12).

\section{Barreras institucionales}

Las barreras institucionales se relacionan con las características de la oferta y la facilidad con la que se accede al tratamiento del $\mathrm{CG}$; pues estas responden a las condiciones en las que se presta el servicio de salud o según régimen de seguridad social; los costos de la atención tanto para las instituciones prestadoras de servicios de salud como para el adulto con CG (23); la pericia médica, entendida como la experiencia del cirujano, su entrenamiento, su edad y el volumen de pacientes atendidos(24); el acceso oportuno a las estrategias de cribado para un diagnóstico temprano del cáncer; la concentración desigual de médicos y de la disponibilidad tecnológica en grandes ciudades; adicional a la falta de inversión en infraestructura, la disponibilidad de camas hospitalarias y de unidades de radiología, entre otras (24). Lo anterior influye en la atención oportuna, el diagnóstico, tratamiento y los cuidados paliativos, así como en la sobrevida, debido a que si un caso de CG es diagnosticado tempranamente su pronóstico a 5 años está entre el $70 \%$ y el $95 \%$, mientras que el CG avanzado apenas alcanza cifras del $20-30 \%$ en ese mismo tiempo(25-28).

Por ejemplo, un estudio descriptivo realizado en Bogotá, Colombia, demostró que de las 42 instituciones prestadoras de salud 31 brindan algún servicio oncológico, donde la quimioterapia es el tratamiento con más oferta y posee el mayor número de especialistas en comparación con la radioterapia $(13,18)$. Además, existen instituciones que no integran las diversas modalidades de tratamiento para el cáncer, lo cual impone barreras de acceso y limitación en la calidad de la atención $(13,18)$. En conjunto con otras barreras genera la interposición de acciones de tutela, en razón a que los usuarios la perciben como un mecanismo de exigibilidad jurídica en respuesta a la negación de los servicios y a las deficiencias en las expectativas del cuidado de las personas representadas en la percepción de la baja calidad en los tratamientos(13).

\section{Barreras económicas}

Respecto a las limitaciones económicas se pudo establecer su relación con la carencia de dinero del adulto con CG y su familia para afrontar los gastos de la enfermedad; en algunos casos dicha carencia obedece al bajo estrato socioeconómico y en otros responde al hecho de que las necesidades económicas, derivadas de la enfermedad, superan la capacidad adquisitiva del núcleo familiar. La 
situación descrita origina variaciones en el presupuesto familiar, a causa del aumento en los pagos del transporte, necesario para los traslados a las actividades de diagnóstico y tratamiento; el hospedaje y la alimentación, lo que puede conducir al abandono del tratamiento(18). Adicionalmente, la vinculación laboral, según el estado de salud en el que se encuentre, puede actuar como un obstáculo que retrasa o dificulta la realización de un tratamiento a largo plazo(29).

Entre la literatura científica se halló que aumenta el riesgo de desarrollar cáncer cuando el grado de escolaridad y el nivel socioeconómico son bajos (30). De igual manera se revisó la conexión entre el nivel socioeconómico y la supervivencia en tres estudios: uno realizado en una cohorte en Taiwán en 3,396 adultos con CG que recibieron cirugía curativa con o sin terapia adyuvante; el segundo en Australia en 139,234 casos; y el tercero en Corea en 1,671 pacientes, los que permitieron concluir: en el primero los adultos con CG menores de 65 años y con alto nivel socioeconómico tienen un riesgo del $68 \%$ menor de mortalidad que aquellos con un nivel socioeconómico bajo y, en concordancia, el segundo y tercero señalaron que la tasa de mortalidad es mayor en pacientes con bajo nivel socioeconómico. La disminución de la supervivencia se atribuyó a la menor disponibilidad de recursos médicos, pobre acceso a tratamientos endoscópicos por diagnóstico tardío, un ambiente más contaminado, malos hábitos de vida, tratamientos subóptimos, menos apoyo social y pobre actitud hacia la salud(31-33).

Además, cuando el bajo nivel socioeconómico se acompaña de cobertura médica otorgada a través del mecanismo de asistencialismo social (Medical Aid System-Corea) la supervivencia a 5 años es menor (43.9\%) comparada con (64.3\%) bajo la cobertura del National Health Insurance System (NHI), si se tiene en cuenta que los adultos con CG de bajo nivel socioeconómico son diagnósticados en etapas avanzadas de la enfermedad, tienen mayores comorbilidades y estas pueden contribuir a la aparición de complicaciones postoperatorias (34). Esta inequidad se profundiza cuando el acceso a los servicios de salud depende del gasto y estos costos superan la capacidad económica del adulto y su familia, que genera el uso del dinero como vía de satisfacción de las necesidades(35).

Como contrapeso a esta situación, una de las estrategias empleadas para disminuir los costos del tratamiento contra el cáncer considerados directos (medicamentos, visitas al médico, servicios de pruebas de diagnóstico), como los indirectos (la pérdida de días de trabajo y productividad, el alojamiento y los costos de los viajes) se refiere al diagnóstico temprano para evitar la etapa IV y las hospitalizaciones prolongadas, extendido a las acciones preventivas, diagnósticas y terapéuticas (cirugía y radiación). El reto de las reformas emprendidas en los diferentes sistemas de salud en algunos países de América Latina(26), deben responder a los factores que modifican el pronóstico del CG como el estadío del cáncer al momento del diagnóstico, las características de los adultos enfermos, y el proceso de atención de acuerdo al tipo de aseguramiento (36).

Entonces, el primer factor que modifica el acceso directo a la atención está conformado por los determinantes sociales del adulto enfermo (representados en la relación entre el nivel socioeconómico y educativo), junto al tiempo transcurrido para la búsqueda de atención en salud. El segundo factor es el paciente, su estado de salud previo y la presencia de comorbilidades (hipertensión, diabetes mellitus, enfermedades del corazón, entre otras) que disminuyen la probabilidad de recibir una cirugía curativa $(37,38)$; también se incluye la raza, donde los pacientes asiáticos tienen mayor sobrevida a los 3 años, seguida por los negros, los hispanos y blancos(39-41). Y el tercero, es la capacidad de pago como requisito para el acceso a las diferentes modalidades de tratamiento que se ofrecen(36).

\section{Barreras culturales}

Las barreras culturales se relacionan con las creencias, costumbres, actitudes, valores, bajo nivel educativo y etnia, los cuales favorecen la búsqueda tardía de atención, frente a la presencia de la alteración del estado de salud(25), añadido a la construcción de significados y percepciones respecto al cáncer, su prevención, cribado y tratamiento, se presentan cuando la familia oculta el diagnóstico al adulto enfermo y/o cuando ellos solicitan al médico tratante la retención de información acerca de la enfermedad al adulto que la padece (26). La situación expuesta genera vacíos en la comunicación médico-paciente y configura una relación carente de confianza; adicionalmente, se crean cambios en la percepción del dolor y su manejo, sentimientos de angustia, de adicción a los medicamentos, de preocupación, sufrimiento, pérdida del grupo familiar, tabúes sobre la incurabilidad y la asociación con una muerte cercana(42).

Otro factor que limita la relación médico-paciente es la falta de apoyo social o familiar y, en consecuencia, dificulta la toma de decisiones en consenso familiar (29). De manera que, unido a la falta de información, limitaciones comunicativas entre médico-paciente, pobre interacción y confianza en el profesional de la salud para la toma de decisiones respecto al manejo médico de su enfermedad(43) puede ocasionar estrés psicológico, falta de adherencia a las instrucciones, abandono o negación al tratamiento y otras situaciones enfrentadas por la familia, que en la mayoría de los casos, son inexpertos en el área de la salud y en el manejo de la enfermedad(44).

Dentro de este contexto, una estrategia para disminuir el impacto de las barreras culturales es la creación de intervenciones educativas que promuevan en los pacientes la credibilidad en la respuesta organizada que brinda el sistema de salud y sus actores (médicos y demás profesionales en salud) y la adquisición de conocimientos acerca del cáncer; a fin de favorecer la toma de decisiones conjunta entre el equipo de salud, el paciente y la familia, que permita generar un contexto de apoyo social. En este 
orden de ideas, los médicos necesitan capacitación en el manejo individualizado de la diversidad cultural que incluya el conocimiento del historial familiar de cáncer gástrico, el cuidado, hábitos de vida(45), sin desconocer la importancia del manejo integral del $\mathrm{CG}(29,42)$.

\section{Barreras de conocimiento}

Como se propuso en el apartado anterior, las barreras de conocimiento profundizan la precariedad en la atención del CG. De esta fuente de inequidades se identifican dos actores. Uno es el médico de atención en primer nivel quien, ante la inespecificidad de los síntomas en las primeras etapas de $\mathrm{CG}$, emite otros diagnósticos de menor complejidad y prescribe tratamientos sintomáticos, en algunos casos por tiempo prolongado, sin seguimiento para evaluar su mejoría; sumado a la ausencia de apoyo con métodos diagnósticos como el endoscópico, inmunológico y/o histológico. Dicho factor puede ser el resultado de las limitaciones propias del sistema de salud o la falta de asociación entre el cuadro clínico del adulto y la impresión diagnóstica del CG, que desencadena un diagnóstico tardío y mal pronóstico $(46,47)$. Alternativamente, puede producirse en respuesta a la formación inadecuada de los profesionales en la atención primaria, referencias inapropiadas a la atención secundaria y también falta de contra referencia, por parte de los especialistas al nivel de atención primaria(16).

El otro actor involucrado en el origen de esta limitación es el paciente, quien desconoce la asociación existente entre los síntomas y la posibilidad del CG como diagnóstico; por esta razón, puede tardar en buscar atención, ya que adjudica de explicaciones de la alteración de salud a causas cotidianas, se automedica, niega los síntomas, recurre a tratamientos de medicina alternativa, o a la búsqueda de atención en salud sin seguimiento y adherencia al tratamiento, pese a la persistencia de los síntomas(26).

Al continuar el seguimiento de este fenómeno, en Colombia, el cáncer gástrico es una entidad que requiere tenerse en cuenta por el médico y por el adulto enfermo ante cualquier molestia de origen gastrointestinal, debido a que más del 90\% los casos de CG son diagnosticados en etapas avanzadas (48) y afecta la sobrevida, porque se reconoce que el diagnóstico temprano ha permitido alcanzar supervivencias superiores al $50 \%$ a los 5 años, en pacientes en estadio I(49).

La influencia de las barreras culturales tiene una intensidad alta en personas con bajo nivel educativo. Un estudio en Colombia durante 3 cuatrienios determinó que el riesgo relativo de muerte por cáncer es mayor tanto en hombres como en mujeres con educación primaria comparada con adultos con educación secundaria. Esta tendencia no cambió a pesar del aumento de la cobertura del sistema de salud; por esta razón se identificó que el CG tiene altas tasas de mortalidad por cáncer también asociado al tabaquismo, factores de riesgo ocupacional, condiciones de vivienda y menor cuidado de su salud(50-56).

Frente a estas barreras se ha planteado la educación a la población como una de las posibles estrategias para aumentar el número de consultas ante dispepsia o epigastralgia mayor a 15 días de evolución, para lo cual se requiere no automedicarse y optar por el estudio de los síntomas a través de la endoscopia de vías digestivas y la toma de biopsia con una sensibilidad superior al $95 \%$ para detectar lesiones presuntivas de cáncer gástrico $(46,52)$. No obstante, el bajo acceso a pruebas de cribado para la detección temprana del CG puede depender del bajo nivel educativo, bajos ingresos en el hogar, desempleo, que impactan negativamente en la sobrevida(56).

\section{Conclusión}

El cáncer gástrico es un problema de salud pública que impacta de manera general al mundo y a Colombia en particular. A pesar de que la incidencia general del cáncer es menor en América Latina que en Europa, la mortalidad sigue siendo mayor debido a factores como: el estadío del CG en el diagnóstico; los determinantes sociales del adulto enfermo y su familia; junto a las barreras de acceso para recibir la atención en salud. Las barreras y/o inequidades destacadas son de tipo administrativo, institucional, económico, cultural y de conocimiento. Las respuestas para contrarrestarlas se relacionan con el uso del dinero para realizar pagos de la atención en salud, búsqueda de orientación y ayuda de la familia y terceros. También, se reconoció la interposición de la acción de tutela como una vía para garantizar el derecho a la salud.

En este sentido, el impacto negativo de las barreras en la sobrevida por CG marca un reto para las reformas del sistema de salud, cuya estructura se caracteriza por la fragmentación operativa y la segmentación institucional como en Colombia; ya que los cambios que se realicen deben aumentar la oportunidad de atención según las necesidades del adulto enfermo, promover estrategias para educar poblaciones en riesgo de CG que aborden su prevención, diagnóstico y tratamiento. Sumado al fortalecimiento del talento humano del sector salud en la identificación del CG como impresión diagnóstica y la necesidad de seguimiento de lesiones precursoras.

En Colombia, la formulación e implementación de la Política de Atención Integral en Salud y el Modelo Integral de Atención en Salud genera una alternativa clara y precisa para que los usuarios del sistema reciban atención en salud bajo una red de servicios integrales conformada por todos los agentes del sistema que actúan de manera coordinada, eficiente, continua, con calidad y oportunidad, en condiciones de equidad que apuntan a reducir las disparidades señaladas e impactar positivamente en la sobrevida de los adultos con CG. 


\section{Consideraciones éticas}

Al estudio realizado no le atañen consideraciones éticas relativas a los casos clínicos y a la intervención de población; sin embargo, es preciso mencionar que las autoras no incurren en faltas éticas respecto a las fuentes referenciadas, pues se atienden a las pautas pertinentes de citación y protección de la propiedad intelectual.

\section{Conflicto de intereses}

Los autores manifiestan que no tienen ningún conflicto de interés.

\section{Agradecimientos}

Gracias al Departamento Administrativo de Ciencia, Tecnología e Innovación (Colciencias) que apoyó la realización del estudio.

\section{Financiación}

El Departamento Administrativo de Ciencia, Tecnología e Innovación (Colciencias) brindó apoyo para la realización del estudio consignado en estas páginas.

\section{Referencias}

1. Fahey JW, Stephenson KK, Wallace AJ. Dietary amelioration of Helicobacter infection. Nutr Res 2015;35(6):461-473. Doi: 10.1016/j.nutres.2015.03.001

2. Epplein M, Zheng W, Li H, Peek RM Jr, Correa P, Gao J, Michel A, Pawlita M, Cai Q, Xiang YB, Shu XO. Diet, Helicobacter pylori strain-specific infection, and gastric cancer risk among Chinese men. Nutr Cancer 2014;66(4):550-557. Doi: 10.1080/01635581. 2014.894096

3. Umesawa M, Iso H, Fujino Y, Kikuchi S, Tamakoshi A, JACC Study Group. Salty Food Preference and Intake and Risk of Gastric Cancer: The JACC Study. J Epidemiol 2016;26(2):92-97. Doi:10.2188/jea. JE20150023

4. Gong EJ, Ahn JY, Jung HY, Lim H, Choi KS, Lee JH, et al. Risk factors and clinical outcomes of gastric cancer identified by screening endoscopy: A case-control study. J Gastroenterol Hepatol 2014; 29: 301-9. DOI: 10.1111/jgh.12387

5. Blanco O, Cantillo A, Rivera J. Enfoque actual del cáncer gástrico. Medicas UIS 2013; 26: 59-70.

6. World Health Organization. International Agency for Research on Cancer. GLOBOCAN 2012: Estimated Cancer Incidence, Mortality and Prevalence Worldwide in 2012. Disponible en: http://globocan.iarc.fr/ Pages/fact sheets cancer.aspx. Fecha de acceso: 15 junio 2016
7. Ministerio de Salud y Protección Social. Sistema de Salud en Colombia. [Internet]. Plan nacional para el control del cáncer en Colombia 2012-2020.Bogotá D.C: M a r zo de 2012 . D i s p o n i ble e n : https://www.minsalud.gov.co/sites/rid/Lists/BibliotecaDi gital/RIDE/IA/INCA/plan-nacional-control-cancer2012-2020.pdf

8. Uribe CJ, Osma S, Herrera V. Cancer Incidence and Mortality in the Bucaramanga Metropolitan Area, 20032007. Colomb Médica 2012; 43(4): 290-297.

9. De Vries E, Uribe C, Pardo C, Lemmens V, Van de Poel E, Forman D. Gastric cancer survival and affiliation to health insurance in a middle-income setting. Cancer Epidemiol 2015;39(1):91-96. Doi: 10.1016/j.canep. 2014.10.012

10. Organización Panamericana de la Salud. OPS/OMS. La Salud Pública en las Américas. Nuevos conceptos, análisis de desempeño y bases para la acción. Washington, D.C.: OPS, 2002 Disponible en: http://www1.paho.org/hq/dmdocuments/2010/FESP_S alud Publica en las_Americas.pdf

11. Ministerio de Salud y Protección Social. Sistema de Salud en Colombia. [Internet]. Política de Atención Integral en Salud. Bogotá D.C: Enero de 2016. Disponible en: https://www.minsalud.gov.co/ sites/rid/Lists/BibliotecaDigital/RIDE/DE/modelo-pais2016.pdf

12. Garcia-Subirats I, Vargas-Lorenzo I, Mogollón-Pérez AS, De Paepe P, Ferreira-Da Silva MR, Unger JP, et al. Determinantes del uso de distintos niveles asistenciales en el Sistema General de Seguridad Social en Salud y Sistema Único de Salud en Colombia y Brasil. Gac Sanit 2014;28(6):480-488. Doi: 10.1016/j.gaceta. 2014.05.010

13. Abadía-Barrero CE; Oviedo-Manrique DG. Itinerarios burocráticos de la salud en Colombia: la burocracia neoliberal, su estado y la ciudadanía en salud. Rev Gerenc y Políticas Salud 2010; 9(18): 86-102.

14. García-Subirats I, Vargas I, Mogollón-Pérez AS, De Paepe P, Da Silva MR, Unger JP, et al. Inequities in access to health care in different health systems: a study in municipalities of central Colombia and north-eastern Brazil. Int J Equity Health 2014;13:10. Doi: 10.1186/1475-9276-13-10

15. Arroyave I, Cardona D, Burdorf A, Avendano M. The impact of increasing health insurance coverage on disparities in mortality: health care reform in Colombia, 1998-2007. Am J Public Health 2013;103(3):e100-106. Doi: 10.2105/AJPH.2012.301143

16. Vargas I, Mogollón-Pérez AS, De Paepe P, Ferreira-Da Silva MR, Unger JP, Vázquez ML. Barriers to healthcare coordination in market-based and decentralized public health systems: a qualitative study in healthcare networks of Colombia and Brazil. Health Policy Plan 2016;31(6):736-748. Doi: 10.1093/heapol/czv126

17. Arroyave I, Burdorf A, Cardona D, Avendaño M. Socioeconomic inequalities in premature mortality in Colombia, 1998-2007: the double burden of noncommunicable diseases and injuries. Prev Med 2014; 64:41-47. Doi: 10.1016/j.ypmed.2014.03.018

18. González-Mariño MA. Diagnóstico de instituciones prestadoras de salud con habilitación de servicios oncológicos en Bogotá, Colombia. Rev Gerenc Polit Salud 2012; 11(22): 92-106.

19. Walker GV, Grant SR, Guadagnolo BA, Hoffman KE, Smith BD, Koshy M, Allen PK, Mahmood U. Disparities in stage at diagnosis, treatment, and survival in nonelderly adult patients with cancer according to 
insurance status. J Clin Oncol 2014;32(28):3118-3125. Doi: 10.1200/JCO.2014.55.6258

20. Blankart CR. Does healthcare infrastructure have an impact on delay in diagnosis and survival?. Health Policy 2012;105(2-3):128-137. Doi: 10.1016/j.healthpol. 2012.01.006

21. Messager M, De Steur W, Boelens PG, Jensen LS, Mariette C, Reynolds JV, et al. Description and analysis of clinical pathways for oesophago-gastric adenocarcinoma, in 10 European countries (the EURECCA upper gastro intestinal group - European Registration of Cancer Care). Eur J Surg Oncol 2016;42(9):1432-1447. Doi:10.1016/j.ejso.2016.01. 001

22. Bouvier AM, Sant M, Verdecchia A, Forman D, Damhuis $\mathrm{R}$, Willem-Coebergh $\mathrm{J}$, et al. What reasons lie behind long-term survival differences for gastric cancer within Europe?. Eur J Cancer 2010;46(6):1086-1092. Doi: 10.1016/j.ejca.2010.01.019

23. Murata A, Muramatsu K, Ichimiya Y, Kubo T, Fujino $Y$, Matsuda S. Influence of hospital volume on outcomes of laparoscopic gastrectomy for gastric cancer in patients with comorbidity in Japan. Asian J Surg 2015;38(1):3339. Doi: 10.1016/j.asjsur.2014.04.008

24. Mahar AL, McLeod RS, Kiss A, Paszat L, Coburn NG. A systematic review of the effect of institution and surgeon factors on surgical outcomes for gastric cancer. J Am Coll Surg 2012; 214(5):860-868. Doi: 10.1016/j.jamcollsurg.2011.12.050

25. Arias-Valencia SA. Inequidad y cáncer: una revisión conceptual. Rev Fac Nac Salud Pública 2009; 27(3): 341-348.

26. Goss PE, Lee BL, Badovinac-Crnjevic T, StrasserWeippl K, Chavarri-Guerra Y, St Louis J, et al. La planificación del control del cáncer en América Latina y el Caribe. Lancet Oncol 2013; 14(5): 391-436. Doi: http://dx.doi.org/10.1016/S1470-2045(13)70048-2

27. Viúdez-Berral A, Miranda-Murua C, Arias-De la Vega F, Hernández-García I, Artajona-Rosino A, Díaz-De Liaño A, Vera-García R. Situación actual en el tratamiento del cáncer gástrico. Rev Esp Enferm Dig 2012;104(3):134141. Disponible en: http://dx.doi.org/10.4321/S113001082012000300006.

28. Martínez JW, Moreno GA, Londoño- De los Ríos PA. Tendencia en el reporte de casos de cáncer en Oncólogos del Occidente, Pereira, Colombia. Rev Médica Risaralda 2012; 18(2): 116-121. Doi: http://dx.doi.org/10.22517/25395203.7867

29. Enard KR, Dolan-Mullen P, Kamath GR, Dixon NM, Volk RJ. Are cancer-related decision aids appropriate for socially disadvantaged patients? A systematic review of US randomized controlled trials. BMC Med Inform Decis Mak 2016;16(1):64. Doi: 10.1186/s12911-016-0303-6

30. Lagergren J, Andersson G, Talbäck M, Drefahl S, Bihagen E, Härkönen J, Feychting M, Ljung R. Marital status, education, and income in relation to the risk of esophageal and gastric cancer by histological type and site. Cancer 2016;122(2):207-212. Doi: 10.1002/cncr. 29731

31. Wu CC, Hsu TW, Chang CM, Yu CH, Wang YF, Lee CC. The effect of individual and neighborhood socioeconomic status on gastric cancer survival. PLoS One 2014;9(2):e89655. Doi: 10.1371/journal.pone. 0089655

32. Stanbury JF, Baade PD, Yu Y, Yu XQ. Cancer survival in New South Wales, Australia: socioeconomic disparities remain despite overall improvements. BMC Cancer 2016;16(1):48. doi: 10.1186/s12885-016-2065-z.
33. Kim NY, Oh JS, Choi Y,Shin J, Park EC. Relationship between socioeconomic status and accessibility for endoscopic resection among gastric cancer patients: using National Health Insurance Cohort in Korea: poverty and endoscopic resection. Gastric Cancer 2017;20(1):61-69. Doi: 10.1007/s10120-016-0597-1

34. Jang JS, Shin DG, Cho HM, Kwon Y, Cho DH, Lee KB, Park SS, Yoon J, Jang YS, Kim IM. Differences in the Survival of Gastric Cancer Patients after Gastrectomy according to the Medical Insurance Status. J Gastric Cancer 2013;13(4):247-254. Doi: 10.5230/jgc. 2013.13.4.247

35. Baradaran-Seyed Z, Nedjat S, Yazdizadeh B, Nedjat S, Majdzadeh R. Barriers of clinical practice guidelines development and implementation in developing countries: a case study in Iran. Int J Prev Med 2013;4(3):340-348.

36. Merletti F, Galassi C, Spadea T. The socioeconomic determinants of cancer. Environ Health 2011;10(Suppl 1):1-7. Doi: 10.1186/1476-069X-10-S1-S7

37. Sarfati D, Gurney J, Stanley J, Koea J. A retrospective cohort study of patients with stomach and liver cancers: the impact of comorbidity and ethnicity on cancer care and outcomes. BMC Cancer 2014;14:821. Doi: 10.1186/1471-2407-14-821

38. Morris AM, Rhoads KF, Stain SC, Birkmeyer JD. Understanding racial disparities in cancer treatment and outcomes. J Am Coll Surg 2010;211(1):105-13. Doi: 10.1016/j.jamcollsurg.2010.02.051

39. Lui FH, Tuan B, Swenson SL, Wong RJ. Ethnic disparities in gastric cancer incidence and survival in the USA: an updated analysis of 1992-2009 SEER data. Dig Dis Sci 2014;59(12):3027-3034. Doi: 10.1007/s10620-014-3275-3.

40. Bautista MC, Jiang SF, Armstrong MA, Kakar S, Postlethwaite D, Li D. Significant Racial Disparities Exist in Noncardia Gastric Cancer Outcomes Among Kaiser Permanente's Patient Population. Dig Dis Sci 2015;60(4):984-995. Doi: 10.1007/s10620-014-3409-7

41. Stessin AM, Sherr DL. Demographic disparities in patterns of care and survival outcomes for patients with resected gastric adenocarcinoma. Cancer Epidemiol Biomarkers Prev 2011;20(2):223-233. Doi: 10.1158/1055-9965

42. Kagawa-Singer M, Dadia AV, Yu MC, Surbone A. Cancer, culture, and health disparities: time to chart a new course?. CA Cancer J Clin 2010;60(1):12-39. Doi: 10.3322/caac. 20051

43. Hamel LM, Penner LA, Albrecht TL, Heath E, Gwede CK, Eggly S. Barriers to Clinical Trial Enrollment in Racial and Ethnic Minority Patients With Cancer. Cancer Control 2016;23(4):327-337.

44. Meuter RF, Gallois C, Segalowitz NS, Ryder AG, Hocking J. Overcoming language barriers in healthcare: A protocol for investigating safe and effective communication when patients or clinicians use a second language. BMC Health Serv Res 2015;15(1):371. Doi: 10.1186/s12913-015-1024-8

45. Sin MK, Culturally Related Health Risks: Gastric Cancer and Asian Americans. J Nurse Pract 2013; 9(10): 706707. Doi: http://dx.doi.org/10.1016/j.nurpra.2013.08.021

46. Calvo-BelmarA. Diagnóstico precoz del cáncer gástrico estrategias de prevención secundaria y dificultades del diagnóstico de lesiones precoces. Rev Med Clin Condes 2011;22(4):477-484. Doi: 10.1016/S0716-8640(11) 70453-9

47. García-Carrasco C. Actualización del diagnóstico y tratamiento del cáncer gástrico. Rev Med Clin Condes 
2013;24(4):627-636. Doi: 10.1016/S0716-8640(13) 70201-3

48. Restrepo-Restrepo AF, Riveros-Vega JH. Guias de práctica clinica - GPC para el Cáncer gástrico.[Tesis Especialización] Bogotá D.C: Facultad de Medicina, Departamento de Medicina Interna, División especializada en Medicina Interna, Universidad Nacional de Colombia; 2014. Disponible en: http://www. bdigital.unal.edu.co/12831/1/5599174.2014.pdf

49. Oliveros R, Navarrera LF. Diagnóstico, estadificación y tratamiento del cáncer gástrico en Colombia desde 2004 a 2008 (REGATE -Colombia). Rev Col Gastroenterol 2012; 27(4): 269-274.

50. De Vries E, Arroyave I, Pardo C. Time trends in educational inequalities in cancer mortality in Colombia, 1998-2012. BMJ Open 2016; 6(4):e008985. Doi: 10.1136/bmjopen-2015-008985

51. De Vries E, Arroyave I, Pardo C, Wiesner C, Murillo R, Forman $\mathrm{D}$, et al. Trends in inequalities in premature cancer mortality by educational level in Colombia, 19982007. J Epidemiol Community Health 2015; 69(5):408415. Doi: 10.1136/jech-2014-204650

52. Pasechnikov V, Chukov S, Fedorov E, Kikuste I, Leja M. Gastric cancer: prevention, screening and early diagnosis. World J Gastroenterol 2014;20(38):1384213862. Doi: 10.3748/wjg.v20.i38.13842

53. De la Torre-Bravo A, Hernández-Guerrero A, PenicheGallareta LF, Tamayo-De la Cuesta JL,Valenzuela-De la Cueva VM, Blancas-Valencia JM. Guía de diagnóstico y tratamiento del cáncer gástrico. Diagnóstico. Rev Gastroenterol Mex 2010;75(2):240-242.

54. Gómez-Zuleta M. Paciente con lesión gástrica subepitelial. Rev Col Gastroenterol 2010;25(4):371-378. Disponible en: http://www.scielo.org.co/pdf/rcg/ v25n4/v25n4a09.pdf

55. Allum WH, Blazeby JM, Griffin SM, Cunningham D, Jankowski JA, Wong R. Guidelines for the management of oesophageal and gastric cancer. Gut bmj 2011; 60: 1449-1472. DOI: 10.1136/gut.2010.228254

56. Allum WH, Blazeby JM, GriffinSM, Cunningham D, Jankowski JA, Wong R. Guidelines for the management of oesophageal and gastric cancer. Gut 2011; 60:1449e1472. doi:10.1136/gut.2010.228254

57. Hahm MI, Park EC, Choi KS, Lee HY, Park JH, Park S. Inequalities in adoption of cancer screening from a diffusion of innovation perspective: identification of late adopters. Cancer Epidemiol. 2011;35(1):90-96. Doi: 10.1016/j.canep.2010.08.009 